\title{
Characterization of Two Site-Specific Human Cytosolic Thioredoxin Mutants (Pro-34 to Ala and Val)
}

\author{
Lin Yuan, Young-Joon Cho, ${ }^{\dagger}$ and Hakjung Kim ${ }^{-}$ \\ Deparment of Chemistry and Department of Molecular Biologv. College of Natural Science, Daegu Chiversity, \\ Gyoungsan 712-714, Korea. ${ }^{*}$ E-mail: himialdaegu ack kr \\ Received August 2+. 2009, Accepted October 8, 2009
}

Key Words: Thioredoxin. Thioredoxin reductase. Site-directed mutagenesis

Thioredoxin (Trx) is a small protein performing multifunctional roles in various organisms from bacteria to mammalians. It has been found as a hydrogen donor for ribonucleotide reductase in Escherichia coli. ' In higher organisms. it is involved in various biological functions as a general dithiol-disulfide oxidoreductase. Its basic function is the catalytic reduction of disulfides in proteins. The oxidized Trx is reduced by Trx reductase and NADPH. In addition the various plysiological roles in many organisms have been reported. "They include the hydrogen donor for ribonucleotide reductase and methionine sulfoxide reductase protein disulfide reduction. $\mathrm{H}_{2} \mathrm{O}_{2}$ reduction, regulation of chloroplast photosynthetic enzy'mes and redox regulation of transcription factors. It is also found at different subcellular compartments such as cytosol. nuclei. and mitochondria in higher organisms. Human cy tosolic Trx is composed of 104 amino acids. ${ }^{4}$ All Trxs from various sources have highly conserved three-dimensional structures. "The active site of Trx is unique as shown in Figure 1. It is a protruding loop from the end of the second $\beta$-sheet strand instead of the cleft. which is more comonony the case.

The consensus sequence of the active disulfide bond of the human cytosolic Trx is Trp-Cys-Gly-Pro-Cys between residue$3 \mathrm{l}$ and 35 . This sequence is highly conserved in most Trxs from various sources as shown in Table 1 . Among 72 Trss searched from different sources. Pro-34 is absolutely conserved. The sitedirected mutagenesis method has been a useful tool for the structure-function study of various proteins. ${ }^{6-110}$ To examine the

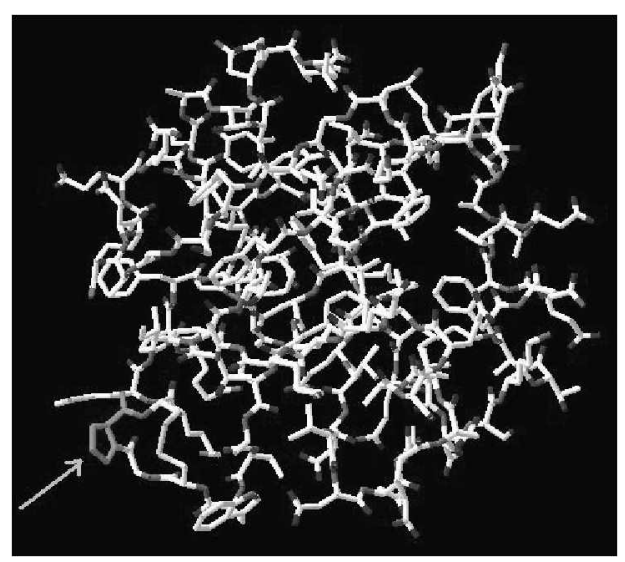

Figure 1. The location of Pro-34 in human cy tosolic thioredoxin. The Pro-34 residue of the active site is indicated by an arrow. importance of this absolutely conserved Pro- 34 residue in human cytosolic Trx function. the Pro- $3+$ residue was mutated to Ala and Val. Site-directed nutagenesis was performed using a mutagenesis kit according to the provided protocol. PCRs were carried out using the human Trx expression vector pPROEX-1: Tr: as a template in a programmable PCR machine with the mutagenic primer shown in Table 2 . The whole DNA sequence of luman Trx coding region was sequenced to verify the integrity of DNA sequences and the mutations. The expressed Trus were purified using a nickel affinity colunm according to the provided protocol. SDS-PAGE revealed that the mutants were highly purified (data not shown).

The quantitative assay method for obtaining steady state kinetic parameters of Tr. activity has not been establishedyet. The most well established assay method for Trx activity is the dithio-

Table 1. Sequence comparison at active site region of thioredoxins from various sources. Among searched 72 thioredosins, 10 thioredoxins are shown here. The Pro-34 residue in human and the corresponding residues in other sources are highlighted in bold italic letters

\begin{tabular}{|c|c|}
\hline Source & Amino acid Sequence \\
\hline Human & ATWCGPCKMI \\
\hline Bos Tamus & ATWCGPCKMI \\
\hline Hits musculus & ATWCGPCKM \\
\hline Drosophila melanogaster (thiol) & ATWCGPCKEM \\
\hline Chlontydia phetmoniae & AEWCGPCKML \\
\hline Escherichia coli & AEWCGPCKM \\
\hline Haemophilus influenzae & APWCGPCKMI \\
\hline Rickettsia cononii & AEWCGPCKML \\
\hline Ancobacteninm tuberculosis & ATWCGPCKMV \\
\hline Fusarium culntorum & ADWCPPCKAI \\
\hline
\end{tabular}

Table 2. Primers for the site-directed mutagenesis. The mismatched bases are underlined

\begin{tabular}{|c|c|}
\hline $\begin{array}{l}\text { Mutations/ } \\
\text { Primers }\end{array}$ & Primer Sequences \\
\hline \multicolumn{2}{|c|}{ Pro-34 to Ala mutation } \\
\hline Sense & 5 -GCCACGTGGTGTGGGGCTTGCAAAATGATCAAG-3 \\
\hline \multicolumn{2}{|c|}{ Antisense 5 -CTTGATCATTTTGCAAGCCCCACACCACGTGCC-3: } \\
\hline \multicolumn{2}{|c|}{ Pro-34 to Val mutation } \\
\hline Sense & 5 -GCCACGTGGTGTGGGGTTTGCAAAATGATCAAG-3 \\
\hline Antisense: & 5'CTTGATCATTTTGCAAACCCCACACCACGTGGC-3' \\
\hline
\end{tabular}


Table 3. Rate of human cy tosolic thioredoxins catalyzed insulin reduction by dithiothreitol and apparent $k_{\text {cad }} / K_{m}$ values of nomal and mutated thioredoxins to rat liver thioredosin reductase

\begin{tabular}{cccc}
\hline Trx & $\begin{array}{c}\text { Time to } \\
\text { precipitation } \\
\text { (Sec) }\end{array}$ & $\begin{array}{c}\text { Rate of } \\
\text { precipitation } \\
\left(\Delta \mathrm{A}_{650} \times \mathrm{min}^{-1}\right)\end{array}$ & $\begin{array}{c}k_{\text {caf }} / K_{m} \\
\left(\mathrm{~min}^{-1} / \mu \mathrm{M}\right)\end{array}$ \\
\hline Normal(Pro-34) & $162 \pm 2$ & $0.153 \pm 0.003$ & $175 \pm 9$ \\
Ala-34 & $186 \pm 5$ & $0.222 \pm 0.006$ & $81 \pm 4$ \\
Val-34 & $215 \pm 14$ & $0.151 \pm 0.008$ & $615 \pm 24$ \\
\hline
\end{tabular}

Values are mean $\pm S$. D. from three separate measurements.

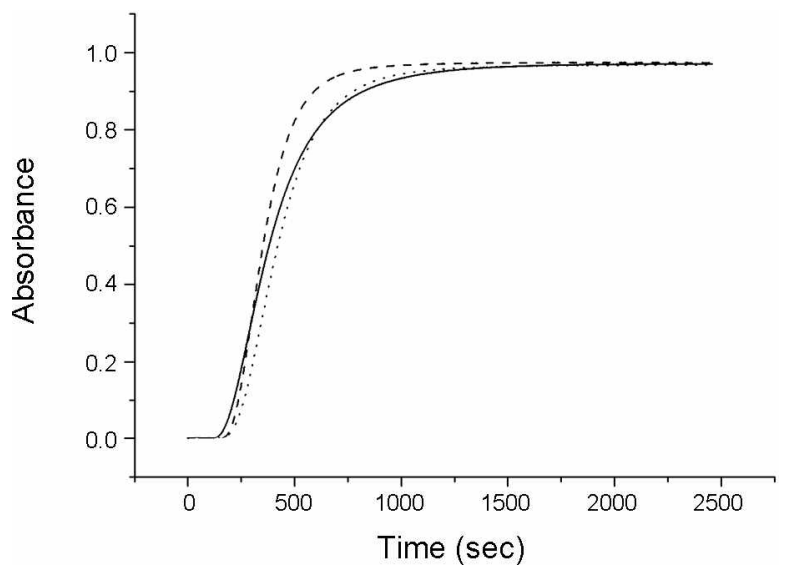

Figure 2. Thioredosin-catalyzed reduction of insulin by dithiothreitol. The absorbance at 650 $117 n$ is plotted against tine. The incubation mixture contained $100 \mathrm{mM}$ potassium phosphate buffer $(\mathrm{pH} 7), 2 \mathrm{mM}$ EDTA, $0.13 \mathrm{mM}$ bovine insulin and $5 \mu \mathrm{M}$ of thioredoxins in a final volume of $500 \mu \mathrm{L}$. Nomal Pro-34 Tri ( solid line), Ala-34 mutant Trx (dashed line) and Val-34 mutant Trx (dotted line).

threitol (DTT)-dependent insulin reduction method. ${ }^{\text {"R }}$ Reduction of insulin by DTT and thioredoxin leads to cleavage of the two interchain disulfide bonds at similar overall rates. As reduction proceeds. a white precipitate is formed mainly from the free $B$ chain of insulin which is insoluble. The time for the complete precipitation of the free insulin $B$ chain aggregates may be long. compared with the rate of the production of free chain resulting in a delay of precipitation as shown in Figure 2. As shown in Figure 2 and Table 3. the activities of the Ala-34 and Val-34 mutants were similar to that of normal Trx. The active sites of enzymes are usually located in a cleft. However, the active site of Trx is unique since it is a protniding loop instead of the cleft as shown in Figure 1. This may contribute to the flexibility of mutations at the residue- 34 in the case of the insulin reduction activity of Tr. The mutations to Ala and Val can be affordable at this site since they are located at the protruding outsider of Trx. Due to the environment with less steric constrains. the mutations at Pro-34 to Ala and Val may not significantly affect the insulin reduction activities of the mutated Tris.

To examine the efficiency of the mutated Trxs as a substrate of mammalian thioredoxin reductase the apparent $k_{\text {cud }} f K_{m}$ values was estimated using rat liver Trx reductase. ${ }^{12}$ The $k_{\text {cad }} / K_{m}$ value of Ala-34 mutant was significantly decreased about 2.2 -fold as shown in Table 3 . This indicated that the mutation to Ala at residue-34 made the Trx a less efficient substrate to its reductase. The decreased side chain volume at residue-34 probably affected the efficient interactions between the $\operatorname{Tr} x$ and its reductase. The $k_{\text {sut }} / K_{m}$ value of Val-34 mutant was significantly increase about 3.5-fold as shown in Table 3. indicating that the mutation to Val at residue-34 made the Trx a more efficient substrate to its reductase. Pro is unique since it is an imino acid. This feature gives an exceptional conformational rigidity compared to other amino acids. The mutation of the Pro-34 to Val may give an additional confornational freedom to Val- 34 mutant to interact more efficiently with the Trx reductase

In this study the effects of the mutations of Pro-34 to Ala and Val on the function of human cytosolic Trx were exanuined using site-directed mutagenesis. the DTT-dependent insulin reduction activity measurement and mammalian Trx reductase assay. The insulin reduction activity of human cytosolic Trx was not significantly affected by the mutations of the Pro-34 to Ala and Val. This result could be related to the nature of the protruding active site of human cytosolic Trx. The $k_{c a t} / K_{m}$ value of Ala-34 mutant to the Trx reductase was significantly decreased. indicating that the mutation of Pro-34 to a smaller amino acid made the Tr $\mathrm{a}$ less efficient substrate to its reductase. This decreased $k_{\text {rat }} / K_{m}$ value of Ala-34 mutant may be due to decreased volume of the side chain. which may result in inefficient interactions between the Ala-34 mutant and the Trx reductase. The Val-34 mutant showed the increase $k_{\text {cum }} / K_{m}$ value to the Trx reductase. indicating that the mutation of Pro-34 to amino acid with similar size and flexibility made the mutant a more efficient substrate. This result indicates that the Pro-34 residue of the Trx should not be mutated to a smaller anino acid to act as a proper substrate to its reductase. The mutation of Pro-34 to amino acid with more conformational freedom and sinular size may help Trx act as a more efficient substrate.

Acknowledgments. The authors thank the 21C Frontier Human Gene Bank for providing a human cytosolic thioredoxin cDNA clone. This research was supported in part by the Daegu University Research Grant. 2009.

\section{Refelences}

1. Laurent, T. C.; Moore, E. C; Reichard, P. J. Biol. Chent. 1964, 239,3436 .

2. Holmgren, A. J. Biol. Chem. 2000, 264, 13963.

3. Amer, E. S. I.: Holmgren, A. Eur. J. Biochem 2000, 267,6102.

4. Wollman, E. E.; d'Auriol, L.; Rimsky, L.; Shaw, A.; Tacquot, T.P.; Wingfield, P, Graber, P.; Dessarps, F.; Robin, P.; Galibert, F.; Bertoglio, T.; Fradelizi, D. J. Biol Chem. 1988, 263, 15506.

5. Ekilund, H.: Gleason, F. K : Holmgren. A. Proteins 1991, $11,13$.

6. Kim, H. Bull. Korean Chem. Soc. 2007, 28, 907.

7. Yoon, M.-Y; Lee, K.-J; Kim, J.; Park, H.-C.; Park, S.-H.; Kim, S. G.; Kim, S.-K.; Choi, T.-D. Bull. Komean Chent. Soc $2009,30,1360$.

8. Koh, J.U.; Cho, H.-Y; Kong, K.-H. Bull. Korean Chem. Soc. 2007, 28.772.

9. Park, S. H.: Kim, B. G.; Lee, S. H.; Lim, Y.: Cheong, Y.; Ahn, J.-H. Bull. Korean Chent. Soc. 2007, 28, 2248

10. Quven, D. V.; Ha, S. C.; Kim, D.; Lee, S.; Park, S.-T.; Kim, K. K; Kim, Y.-G. Bull Korean Chem Soc 2007, 28, 2539

11. Holmgren, A. J. Biol. Chem. 1979, 254, 9627.

12. Gromer, S.; Arscott, L. D.: Williams, Jr., C. H.: Schimer, R. H.; Becker, K. J. Biol Chem. 1998, 273, 20096. 